\title{
A NOTE ON RIESZ SUMMABILITY OF THE TYPE $e^{n^{\alpha}}$
}

\section{FU TRAING WANG}

Recently I proved the following result in the case $r=2$ (Wang [4 $]^{1}$ ).

Let $\sigma_{n}^{(r)}$ be the rth Cesidro mean of the series $\sum_{n=0}^{\infty} a_{n}$. If $\sigma_{n}^{(r)}-s=o\left(n^{-r \alpha}\right)$ $0<\alpha<1$, as $n \rightarrow \infty$, where $r$ is a positive integer, and $a_{n}>-K n^{\alpha-1}$, the series converges to sum $s$.

For the case $r=1$ this result has been established by Boas [1]. His argument, however, does not seem to be applicable in any simple way to the general case.

The object of this note is to prove a theorem on Riesz summability of type $e^{n^{\alpha}}$, and then to deduce the result above from a Tauberian theorem of Hardy [2].

Let us put $C_{\tau}(\omega)=a_{0} e^{\tau \omega^{\alpha}}+\sum_{n<\omega}\left(e^{\omega^{\alpha}}-e^{n^{\alpha}}\right)^{\tau} a_{n}$. A series $\sum_{n=0}^{\infty} a_{n}$ is said to be summable $\left(e^{n^{\alpha}}, \tau\right)$ to the sum $s$ if

$$
C_{\tau}(\omega)=s e^{\tau \omega^{\alpha}}+o\left(e^{\tau \omega^{\alpha}}\right) \text {. }
$$

The result by Hardy which is to be called upon is the following: If the series $\sum_{n=0}^{\infty} a_{n}$, with terms $a_{n} \geqq-K n^{\alpha-1}, 0<\alpha<1$, is summable $\left(e^{n^{\alpha}}, \tau\right)$, it is convergent. We shall now prove the following theorem.

THEOREM. If $\sigma_{n}^{(r)}-s=o\left(n^{-r \alpha}\right), 0<\alpha<1$, as $n \rightarrow \infty$, the series $\sum_{n=0}^{\infty} a_{n}$ is summable $\left(e^{n^{\alpha}}, \tau\right)$ to the sum $s$, where $\tau>r /(1-\alpha)$.

To prove this let $\beta_{n}=\left(e^{\omega^{\alpha}}-e^{n^{\alpha}}\right)^{\tau}, \Delta \beta_{n}=\beta_{n}-\beta_{n+1}, \Delta^{r+1} \beta_{n}=\Delta^{r} \beta_{n}$ $-\Delta^{r} \beta_{n+1}$ and

$$
s_{n}^{(r)}=\sum_{\nu=0}^{n}\left(\begin{array}{c}
n-\nu+r \\
n-\nu
\end{array}\right) a_{\nu}
$$

$m=[\omega]$. Then, by successive Abel's transformations we have

$$
\begin{aligned}
C_{\tau}(\omega) & =a_{0} e^{\tau \omega \alpha}+\sum_{n=1}^{m} \beta_{n} a_{n} \\
& =a_{0} e^{\tau \omega \alpha}+\sum_{n=1}^{m-r+1} s_{n}^{(r)} \Delta^{r+1} \beta_{n}+\sum_{i=0}^{r} s_{m-i}^{(i)} \Delta^{i} \beta_{m-i}-\sum_{i=0}^{r} s_{0}^{(i)} \Delta^{i} \beta_{1} \\
& =a_{0} e^{\tau \omega \alpha}+J_{1}+J_{2}-J_{3} .
\end{aligned}
$$

Received by the editors January 6, 1944.

${ }^{1}$ Numbers in brackets refer to the references listed at the end of the paper. 
Since $\beta_{m-i}=\left(e^{\omega^{\alpha}}-e^{(m-i)^{\alpha}}\right)^{r}=O\left(e^{\tau \omega^{\alpha}} \omega^{\tau(\alpha-1)}\right)$, it follows that

$$
\Delta^{(i)} \beta_{m-i}=O\left(e^{\tau \omega^{\alpha}} \omega^{\tau(\alpha-1)}\right) \quad \text { for } 0 \leqq i \leqq r .
$$

By a familiar theorem on Cesàro sums we get

$$
s_{m-i}^{(i)}=O\left(\omega^{r}\right), \quad \text { for } 0 \leqq i \leqq r,
$$

and from this

$$
J_{2}=O\left(e^{\tau \omega^{\alpha}} \omega^{\tau(\alpha-1)+r}\right)=o\left(e^{\tau \omega^{\alpha}}\right) .
$$

Since $\Delta^{i} \beta_{1}=O\left(e^{i \omega^{\alpha}} \omega^{i(\alpha-1)}\right)$, for $1 \leqq i \leqq r$, and $\beta_{1}=\left(e^{\omega^{\alpha}}-e\right)^{\tau}, s_{0}^{(i)}=s_{0}$ $=a_{0}$ we get

$$
J_{3}=e^{\tau \omega^{\alpha}} a_{0}+o\left(e^{\tau \omega^{\alpha}}\right) .
$$

By the hypothesis of the theorem we have

$$
\begin{aligned}
J_{1}= & \sum_{n=1}^{m-r-1} s_{n}^{(r)} \Delta^{r+1} \beta_{n}=s \sum_{n=1}^{m-r-1}\left(\begin{array}{c}
n+r \\
n
\end{array}\right) \Delta^{r+1} \beta_{n} \\
& +o\left(\sum_{n=1}^{m-r-1} n^{r(1-\alpha)}\left|\Delta^{r+1} \beta_{n}\right|\right) .
\end{aligned}
$$

It follows by mathematical induction that

$$
\Delta^{r+1} \beta_{n}=(-1)^{r+1} \int_{n}^{n+1} d x_{1} \int_{x_{1}}^{x_{1}+1} d x_{2} \cdots \int_{x_{r}}^{x_{r}+1} B^{(r+1)}\left(x_{r+1}\right) d x_{r+1},
$$

where

$$
\beta^{(r+1)}(x)=\frac{d^{r+1}}{d x^{r+1}}\left\{\left(e^{\omega^{\alpha}}-e^{x^{\alpha}}\right)^{\tau}\right\} .
$$

By direct differentiation we have

$$
B^{(r+1)}(x)=\sum_{j=1}^{r+1} \sum_{i=1}^{r} c_{i j} e^{(r-j) \omega^{\alpha}} e^{j x^{\alpha}} x^{i \alpha-r-1},
$$

where $\tau$ is taken as a positive integer and the constants $c_{i j}$ depend upon $i, j, \tau, r, \alpha$. Hence we get

$$
\Delta^{r+1} \beta_{n}=\sum_{i=1}^{r} O\left(e^{(\tau-i) \omega^{\alpha}} e^{i n^{\alpha}} n^{(r+1)(\alpha-1)}\right) .
$$

It is easily verified by Abel's transformation that

$$
\sum_{n=1}^{m-r-1}\left(\begin{array}{c}
n+r \\
n
\end{array}\right) \Delta^{r+1} \beta_{n}=e^{\tau \omega^{\alpha}}+o\left(e^{\tau \omega^{\alpha}}\right) \text {. }
$$


Hence by (5), (6), and (7)

$$
\begin{aligned}
J_{1} & =s e^{\tau \omega^{\alpha}}+o\left(e^{\tau \omega^{\alpha}}\right)+o\left(\sum_{i=1}^{\tau} \sum_{n=1}^{m-r-1} e^{(\tau-i) \omega^{\alpha}} e^{i n^{\alpha}} n^{\alpha-1}\right) \\
& =s e^{\tau \omega^{\alpha}}+o\left(e^{\tau \omega^{\alpha}}\right) .
\end{aligned}
$$

The proof of the theorem follows from (4), (2), (3), and (8).

I conclude by observing that the theorem is the best possible of its kind (Wang [4]).

\section{REFERENCES}

1. R. P. Boas, Tauberian theorem for (C.1) summability. Duke Math. J. vol. 4 (1938) pp. 227-230.

2. G. H. Hardy, An extension of a theorem on oscillating series, Proc. London Math. Soc. (2) vol. 12 (1913) pp. 174-180.

3. G. H. Hardy and M. Riesz, The general theory of Dirichlet series, 1914.

4. F. T. Wang, Some remarks of oscillating series, Quarterly J. Math.

National University of Chekiang 